\title{
Ultrasonic Mammography with Circular Transducer Array
}

\author{
Krzysztof J. OPIELIŃSKI, Piotr PRUCHNICKI, Tadeusz GUDRA \\ Chair of Acoustics and Multimedia, Department of Electronics, Wroctaw University of Technology \\ Wybrzeże Wyspiańskiego 27, 50-370 Wrocław, Poland; e-mail: krzysztof.opielinski@pwr.wroc.pl
}

(received November 13, 2013; accepted September 30, 2014)

\begin{abstract}
Ultrasonic projection imaging is similar to X-ray radiography. Nowadays, ultrasonic projection methods have been developed in the set-up of multi-element flat arrays with miniature transducers, where one of the array acts as a transmitter and the other one is a receiver.

In the paper, a new method of the projection imaging using a 1024-element circular ultrasonic transducer array is presented. It allows the choice of a projection scanning plane for any angle around a studied object submerged in water. Fast acquisition of measurement data is achieved as a result of parallel switching of opposite transmitting and receiving transducers in the circular array and vertical movement of the array. The algorithm equalizing the length of measurement rays and the distances between them was elaborated for the reconstruction of projection images.

Projection research results of breast phantom obtained by means of the elaborated measurement setup and compared with mammography simulations (acquired through overlapping of X-ray tomographic images) show that ultrasonic projection method presented in this paper (so-called ultrasonic mammography) can be applied to the woman's breast and be used as a diagnosis for an early detection of cancerous lesions. It can, most of all, be used as an alternative or complementary method to standard mammography, which is harmful because of ionizing radiation and invasive due to the mechanical compression of tissue.
\end{abstract}

Keywords: ultrasonic projection imaging (UPI), ultrasonic mammography, circular transducer array, woman's breast tissue.

\section{Introduction}

Ultrasonic projection method is analogous to roentgenography (RTG imaging) (ERMERT et al., 2000; OpIELINSKI, 2012; OpIELINSKI, GUdRA, 2005; REGUIEG et al., 2006). Compared to RTG it has an essential advantage of no ionizing radiation, which means that in vivo biological structures and especially human tissues can be examined multiple times from various selected directions. In case of a source generating a plane wave (parallel beam rays), a parallel projection image will be obtained (orthogonal projection). If a source generating a spherical ultrasonic wave is used (divergent beam rays), it will be an image in central projection. It is also possible to use a cylindrical wave source for central-parallel projection (beam rays are divergent in one plane and parallel in a perpendicular plane).

The effects associated with propagation of ultrasonic waves in biological structures (scattering, diffraction, interference, refraction and reflection) cause slight deformations of the projection image, provided the local values of ultrasound velocity are not significantly diversified which is a condition that is met for most soft tissues (DUCK, 1990; KAK, SLANEY, 1988). Using the projection method (transmission), it is also possible to get twice the level of amplitude of ultrasonic received pulses in comparison to echo method (OPIELIŃsKI, 2011). On the other hand projection method requires access to the studied medium from two opposite directions and uses liquid coupling (usually water).

Ultrasonic projection images of the studied medium from various directions make it possible to perform three-dimensional estimation of the type and position of heterogeneities in it. Additionally, it is possible to use projection imaging in relation to several acoustic parameters which are digitally determined on the basis of information obtained directly from ultrasonic pulses propagating through a biological structure (amplitude, runtime, mid frequency downshift, received pulse spectrum). Based on those parameters, it is possible to obtain several various ultrasonic projection 
images, each of which defines slightly different structure features (OpIELINSKI, 2012) (e.g. distribution of mean (projected) values of attenuation coefficient and ultrasonic wave propagation velocity, derivative of attenuation coefficient in relation to frequency, nonlinear acoustic parameter $B / A$ ). Such complex projection characteristics can be of significant importance in the process of detecting and diagnosing cancerous lesions in soft tissue, especially in women's breasts (DUCK, 1990; LANDini et al., 1985; OpIELINSKI, GUdRA, 2013; OPIELIŃSKI et al., 2013; YANG et al., 1991).

Ultrasonic projection imaging is qualitative, which means it is not as good as quantitative imaging using ultrasonic transmission tomography method (DURIC et al., 2007; OpIELIŃSKI, 2011; OPIELINSKI, GUDRA, 2004). However, ultrasonic projection is a more cost effective solution. Additionally, fast acquisition of measurement data makes it possible to obtain images in pseudo-real time, with minor delay resulting from buffering (ultrasonic transmission camera (ERMERT et al., 2000; OpIELINSKI, 2012; OpIELINSKI et al., 2011; REGUieg et al., 2006)).

The following paper presents an innovative method for ultrasonic projection imaging of female breast tissue, using a circular ultrasonic transducer array (GUDRA, OPIELINSKI, 2006) and specially designed algorithms that transform measurement data associated with parallel projection rays of different length and distances between one another (chords) to data associated with rays of equal length and distance. By analogy to $\mathrm{X}$-ray mammography, this method can be called ultrasonic mammography. Its significant advantages are the following: short examination time with high scanning resolution, sufficient ultrasonic wave intensity with an option but no obligation to focus the beam, no uncomfortable mechanical compression of breast, fast selection of many various projection scanning planes around the medium and the option to perform multiple in vivo examinations without exposing the patient to harmful ionizing radiation.

\section{Ultrasonic projection with a circular transducer array}

Mechanical movement of a pair of single-element ultrasonic probes (transmitting and receiving) makes it possible to adjust the scanning resolution and match ultrasonic transducers with the examined structure which produces images of the best quality (Fig. 1). Unfortunately, long measurement time means that this method cannot be used for in vivo examinations (Opielinski, Gudra, 2005).

Projection visualisation of biological media in pseudo-real time (ultrasonic transmission camera) can be performed using 2-D flat ultrasonic arrays (Fig. 2). However, the design of such arrays is very complex and expensive due to small sizes of elementary transducers, the required repeatability of the parameters of the arrays, switching and the way electrodes are attached (Drinkwater, Wilcox, 2006; Eames, HosSACK, 2008; Green et al., 1974; Karaman et al., 2009; Kim, Song, 2006; Opieliński et al., 2010a; OPIELIŃSKI et al., 2010b; WYGANT et al., 2006).

In order to increase scanning resolution, the sizes of array transducers and the distances between them should be as small as possible. This results in decreasing the intensity of the ultrasonic wave generated in the studied medium and makes it necessary to use two-dimensional phase focusing, synthetic apertures or mechanical lenses (Brettel et al., 1981; Chiao, Thomas, 1996; Granz, Oppelt, 1987; Hoctor, KASSAM, 1990; Johnson et al., 2005). Various specialists have been developing such cameras for

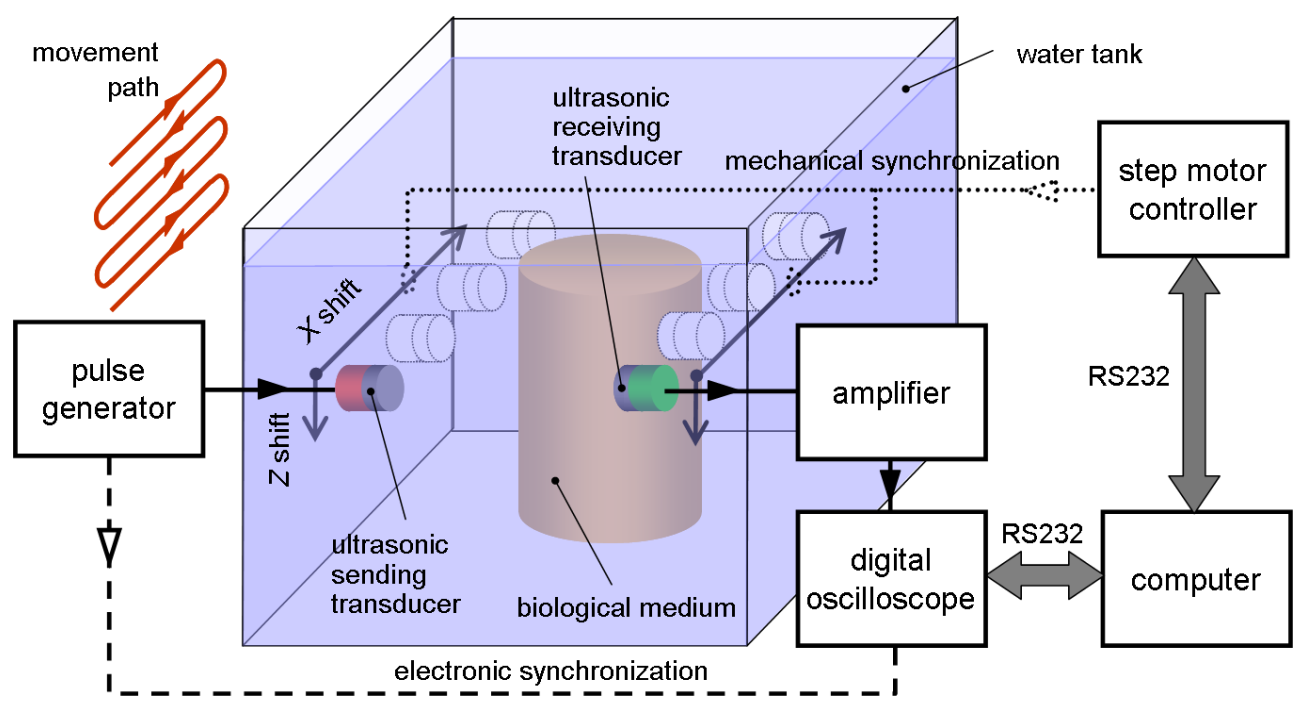

Fig. 1. Parallel projection with a single pair of ultrasonic transducers. 


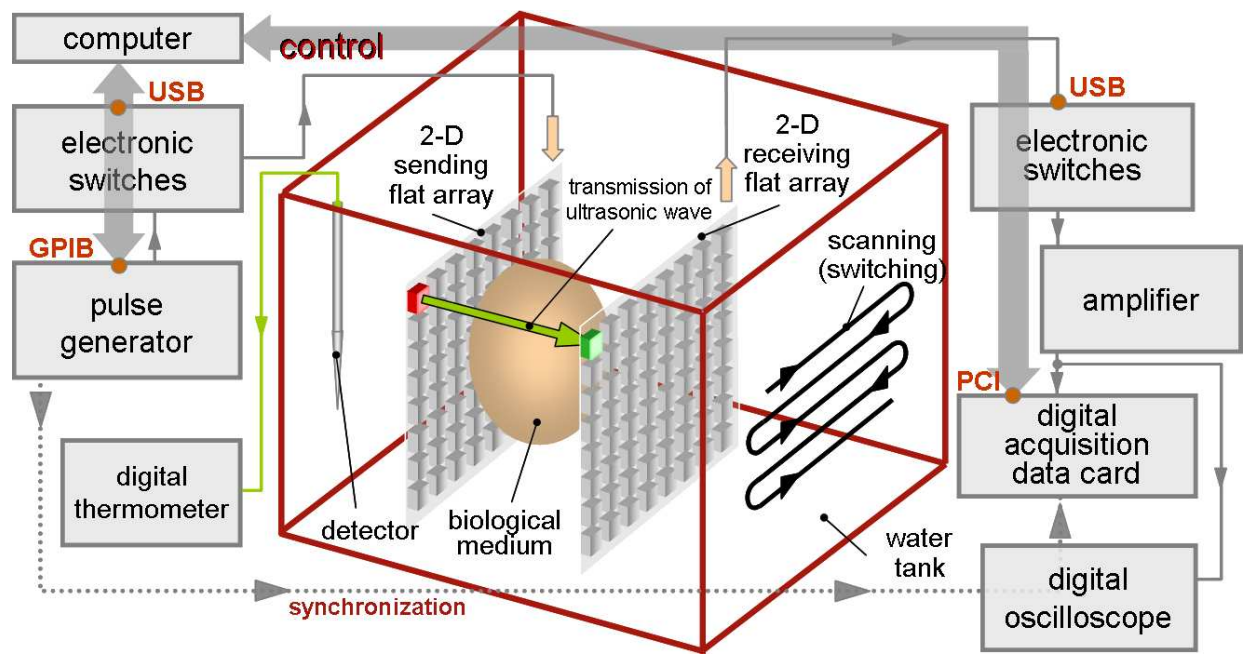

Fig. 2. Parallel projection with a pair of flat ultrasonic transducer arrays.

a long time in several well known scientific centres around the world (BRETTEL et al., 1981; ERMERT et al., 2000; Granz, Oppelt, 1987; Green et al., 1974; Reguieg et al., 2006). One of such cameras is also being worked on by the authors of this paper in the Ultrasonic Technology Laboratory of the Department of Electronics in Wroclaw University of Technology (OPIELINSKI, 2012; OPIELINSKI, GUDRA, 2010; 2013; OPIELINSKI et al., 2011).

This work presents the innovative method of ultrasonic projection imaging using a circular ultrasonic transducer array (GUDRA, OPIELINSKI, 2006) intended for in vivo visualisation of the internal structure of women's breast tissue (Fig. 3).

This method is a compromise between cost effective, precise, universal but time consuming projection by means of mechanically moved pair of single-element probes, and an expensive, more complex and less precise but fast ultrasonic transmission camera with electronic switching of elementary transducers in flat twodimensional arrays.

Initial ultrasonic projection imaging examinations using a 1024-element circular ultrasonic transducer array were performed on the measurement stand for ultrasonic transmission tomography (OPIELIŃSKI et al., 2013) by selecting parallel projections (for the chosen projection planes) from a three-dimensional set of tomographic data in divergent beam geometry (acquired during measurements of biopsy CIRS Model 052A breast phantom), and by appropriate data transformation. As a part of this study a measurement set-up with a circular ultrasonic transducer array was modified to achieve faster object scanning using parallel ultrasonic projection (Fig. 3). Data acquisition on

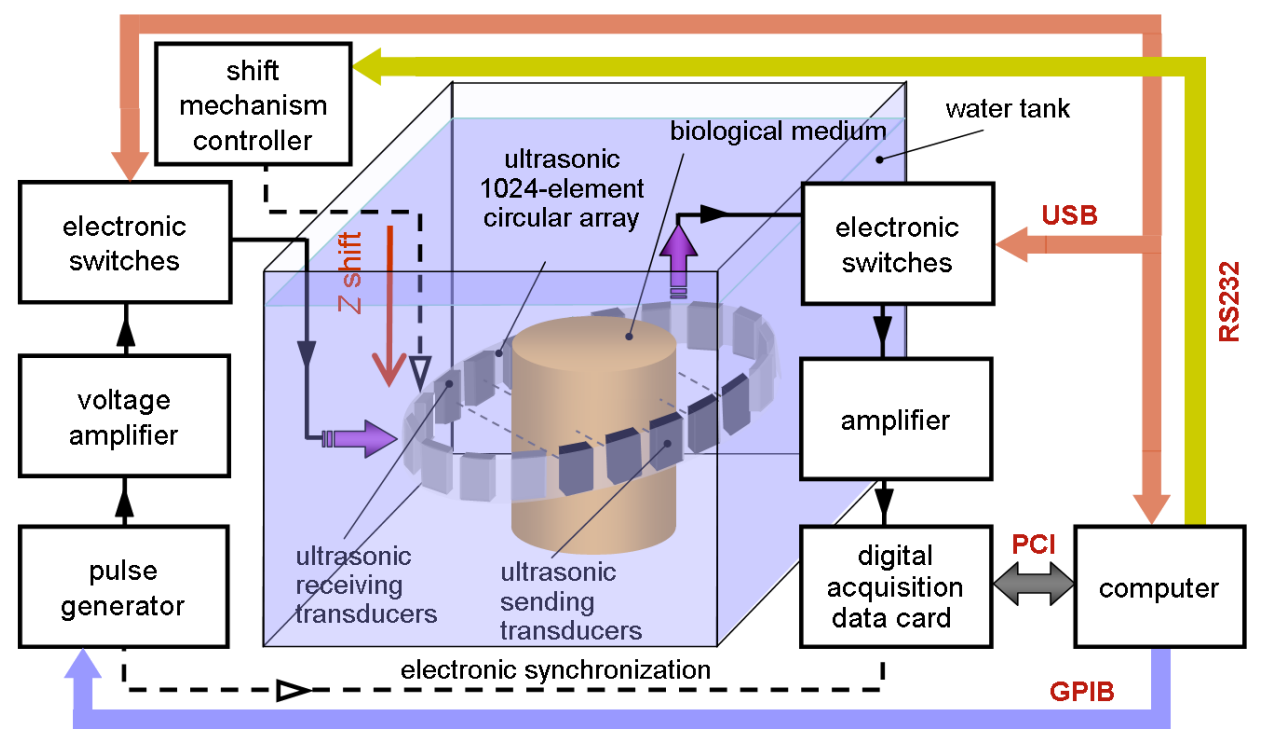

Fig. 3. Block diagram of the measurement set-up for parallel ultrasonic projection using a 1024-element circular ultrasonic transducer array. 
the measurement stand is realised in parallel-ray geometry (KAK, Slaney, 1988). For the selected projection (scanning) plane suitable pairs of elementary transducers in the array ring are switched in parallel in a transmission-reception sequence for every position of the vertically moved (mechanically) ring (Fig. 4).

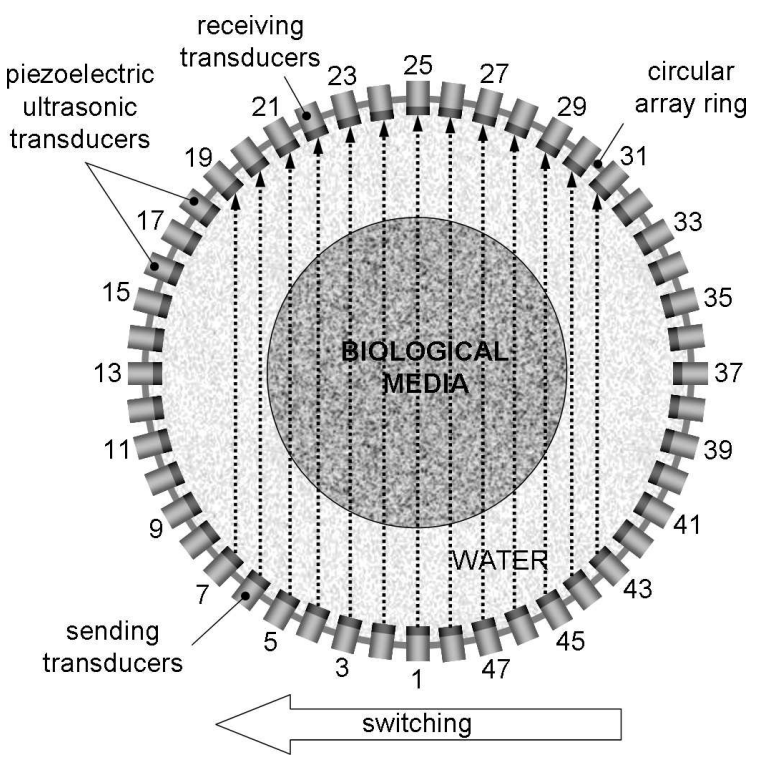

Fig. 4. Example-projection scanning sequence for transmitting and receiving ultrasonic transducers of a 48-element circular array (the first transmitting transducer in the sequence - no. 43, the last transmitting transducer in the sequence - no. 7).

The scanning planes are selected by defining the first and last transmitting transducers in scanning sequences with an odd number. The middle transducer of such a sequence determines the diameter of the array and the direction of projection (Fig. 4). Transmitting-receiving transducers of a circular array (width $0.5 \mathrm{~mm}$, height $18 \mathrm{~mm}$, distance between edges $0.2 \mathrm{~mm}$, operating frequency $\sim 2 \mathrm{MHz}$, wave length in soft tissue $\lambda=0.77 \mathrm{~mm}$, internal diameter of the ar- ray $260 \mathrm{~mm}$ ) are activated during transmission from a generator by several cycles of rectangular pulses via a voltage amplifier system and a closed electronic switch. The signals passing through the studied biological medium are received by means of a closed electronic switch and a low noise amplifier. The received signals are recorded on a computer hard drive (using a computer card for acquisition of signals with sampling frequency of $30 \mathrm{MHz}$ ) which, by means of suitable software with measurement algorithms for acoustic parameters of ultrasonic pulses, are used to directly determine 3 projection signal parameters: amplitude $A_{L_{n}}$, runtime $t_{L_{n}}$ and frequency $f_{L_{n}}$. The method of associating those parameters with projections of distributions of local acoustic parameter values - attenuation coefficient, propagation velocity of ultrasonic wave and derivative of attenuation in relation to frequency in the examined section plane of the biological medium respectively, was defined using the following formulas (Fig. 5):

$$
\begin{aligned}
A_{L_{n}} & =\frac{A_{o}}{e^{\left[\int_{L_{n}} \frac{1}{\mathrm{~d} l_{n}}\left(\ln \left(\frac{A\left(l_{n(i)}\right)}{A\left(l_{n(i+1)}\right)}\right)\right) \mathrm{d} l_{n}\right]}} \\
& =\frac{A_{o}}{e^{\left[\int_{L_{n}}\left(\alpha\left(x, y, f_{o}, T_{o}\right)\right) \mathrm{d} l_{n}\right]}} \\
t_{L_{n}} & =\int_{L_{n}} \mathrm{~d} t_{L_{n}}=\int_{L_{n}} \frac{\mathrm{d} t_{L_{n}}}{\mathrm{~d} l_{n}} \mathrm{~d} l_{n}=\int \frac{1}{c\left(x, y, T_{o}\right)} \mathrm{d} l_{n}, \\
f_{L_{n}} & =f_{o}-2 \sigma^{2} \int_{L_{n}} \alpha_{o}\left(x, y, T_{o}\right) \mathrm{d} l_{n},
\end{aligned}
$$

where $L_{n}$ - array ring chord connecting the transmitting and receiving transducer pair, $n$ - chord number, $\mathrm{d} l_{n}$ - infinitely short sections of fractional paths of a wave along the chord $L_{n}, \mathrm{~d} t_{n}$ - infinitely short times of wave passage along the chord $L_{n}, \alpha\left(x, y, f_{o}, T_{o}\right)-$

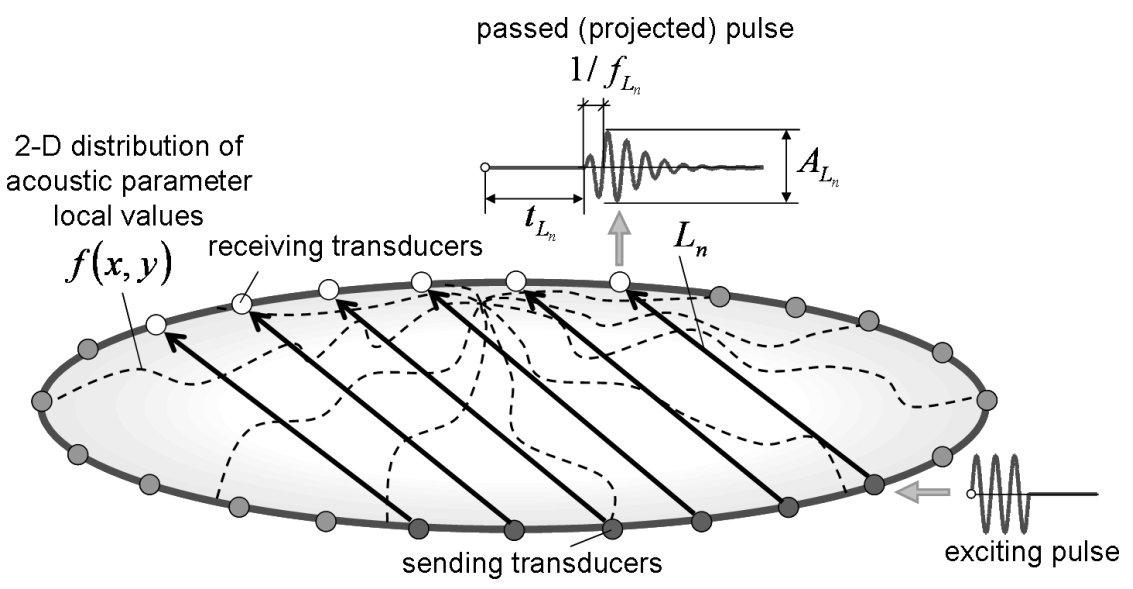

Fig. 5. Representation of ultrasonic projection along parallel chords of a two-dimensional function of acoustic parameter distribution determined on a circular plane. 
local value of ultrasonic wave attenuation coefficient on a plane (for $z=z_{o}$ ) in a point represented by coordinates $(x, y)$ for a set temperature $T_{o}$ and frequency $f_{o}, c\left(x, y, T_{o}\right)$ - local value of propagation velocity of ultrasonic wave on a plane (for $z=z_{o}$ ) in a point represented by coordinates $(x, y)$ for a set temperature $T_{o}, \alpha_{o}\left(x, y, T_{o}\right)=\partial \alpha\left(x, y, f_{o}, T_{o}\right) / \partial f-$ local value of derivative of ultrasonic wave attenuation coefficient in relation to frequency on a plane (for $z=z_{o}$ ) in a point represented by coordinates $(x, y)$ for a set temperature $T_{o}$, in a point $f=f_{o}, A_{o}$ - amplitude of the transmitted ultrasonic pulse (near the surface of the transmitting transducer in water), $A\left(l_{n(i)}\right)$ and $A\left(l_{n(i+1)}\right)$ - amplitudes of ultrasonic pulse after it propagates along paths $l_{n(i)}$ and $l_{n(i+1)}$ on chord $L_{n}$, where $\left(l_{n(i+1)}-l_{n(i)}\right)=d l_{n}, \sigma^{2}$-variance of ultrasonic pulse power in distilled water.

Since the scanning was performed inside the ring of the circular array, the measurement data do not directly represent parallel projection. It is necessary to transform them in order to equalise the lengths (Fig. 6a) and mutual distances (Fig. 6b) of the measurement rays associated with them.

a)

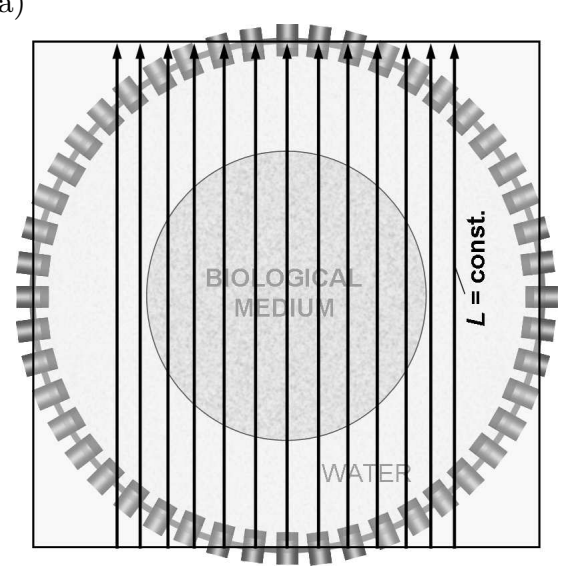

b)

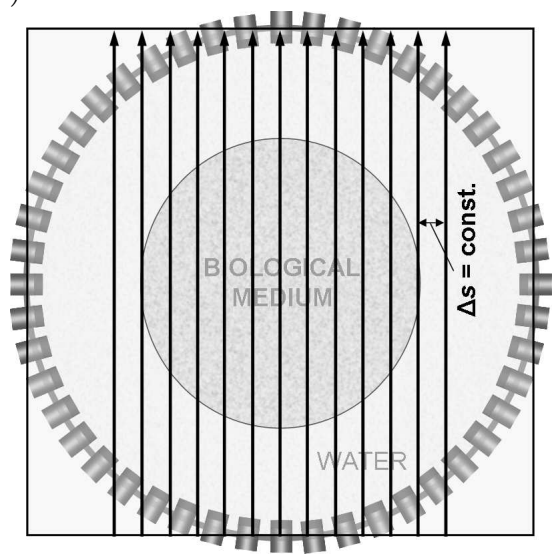

Fig. 6. Graphical representation of equalising lengths (a) and distances (b) of measurement rays associated with specific projection data.
In order to equalise the length of measurement rays from the value of chord $L_{n}$ to the value of inside diameter $2 R_{o}$ on the array ring, the following transformations of direct projection values of signal parameters were used:

$$
\begin{aligned}
& A_{2 R_{o}}\left(L_{n}\right)=A_{L_{n}} \cdot e^{\left(L_{n}-2 R_{o}\right)} \cdot \alpha_{w}, \\
& t_{2 R_{o}}\left(L_{n}\right)=t_{L_{n}}+t_{w\left(2 R_{o}-L_{n}\right)}=t_{L_{n}}+\left(\frac{2 R_{o}-L_{n}}{c_{w}}\right), \\
& f_{2 R_{o}}\left(L_{n}\right)=f_{o}-f_{w\left(L_{n}\right)}+f_{L_{n}}-\left(f_{o}-f_{w\left(L_{n}\right)}\right) \cdot \frac{2 R_{o}}{L_{n}},
\end{aligned}
$$

where $\alpha_{w}$ - value of ultrasonic wave attenuation coefficient in distilled water in the measurement conditions, $c_{w}$ - value of propagation velocity of ultrasonic wave in distilled water in the measurement conditions, $f_{w\left(L_{n}\right)}$ - centre frequency of the ultrasonic wave after it propagated through distilled water along chord $L_{n}$. The length of individual chords is determined using the following formula:

$$
L_{n}=2 R_{o} \cdot \sin \left(\frac{\left|n_{T}-n_{R}\right| \cdot \pi}{N_{t a}}\right),
$$

where $N_{t a}$ - the number of transducers in the circular array, $n_{T}=1,2, \ldots, N_{t a}$ - the number of transmitting transducer, $n_{R}=1,2, \ldots, N_{t a}$ - the number of receiving transducer which is appropriately paired with the transmitting one (Fig. 4). It is also possible to indirectly equalise the measurement rays by transforming projection values of acoustic parameters:

$$
\begin{aligned}
\alpha_{p 2 R_{o}}\left(L_{n}\right) & =\frac{1}{L_{n}} \ln \left(\frac{A_{w\left(L_{n}\right)}}{A_{L_{n}}}\right)+\alpha_{w}, \\
c_{p 2 R_{o}}\left(L_{n}\right) & =\left(\frac{2 R_{o} \cdot c_{w}}{t_{L_{n}} \cdot c_{w}-L_{n}+2 R_{o}}\right), \\
\alpha_{o_{p 2 R_{o}}}\left(L_{n}\right) & =\frac{1}{2 \sigma^{2}} \cdot\left(\frac{f_{o}-f_{w\left(L_{n}\right)}}{L_{n}}+\frac{f_{w\left(L_{n}\right)}-f_{L_{n}}}{2 R_{o}}\right),
\end{aligned}
$$

where $A_{w\left(L_{n}\right)}$ is the amplitude of ultrasonic wave pulse after it propagated through distilled water along chord $L_{n}$. As a result ultrasonic projection images can be presented in two ways: as pixels representing direct projection values of signal parameters $((4),(5),(6))$ or indirect projection values of acoustic parameters $((8)$, (9), (10)).

In order to equalise the distances between the measurement rays, a fixed scanning step $\Delta s$ (Fig. 6b) was used as the shortest distance between outermost measurement chords (Fig. 7a):

$$
\Delta s=L_{n_{\max }} \cdot \sin \left(\frac{\pi}{N_{t a}}\right),
$$

where $L_{n_{\max }}$ is the length of the outermost measurement chord. Projection values, that had been previ- 
a)

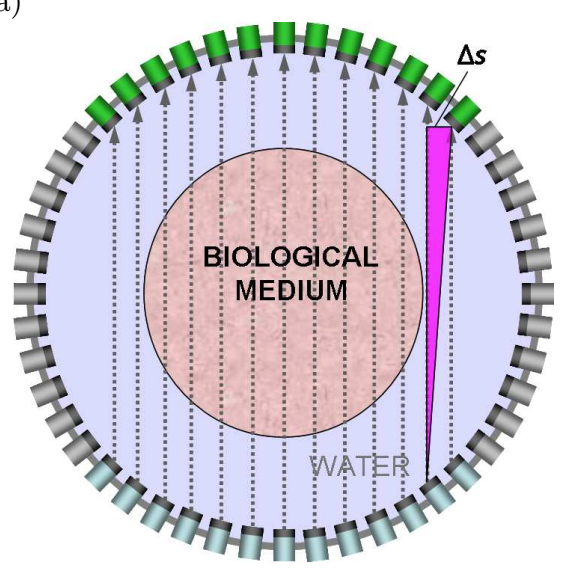

b)

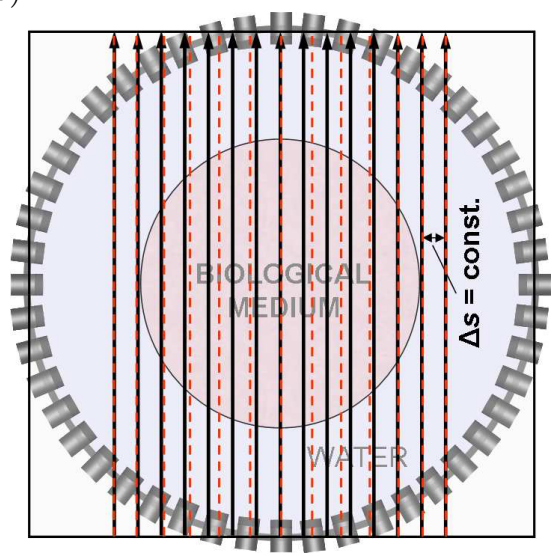

Fig. 7. The method of equalising distances between measurement rays associated with appropriate projection data: a) determining the scanning step, b) the shift of measurement rays with interpolation of projection values.

ously equalised in the aspect of the length of measurement rays, were linearly interpolated for all new positions (Fig. 7b) mutually distanced by a fixed step value of $\Delta s$. The size used for the square pixel grid of projection images was $\Delta s$.

In order to allow for non-parallelism of surfaces for transmitting and receiving transducer pairs located on the chords of the array around its diameter, it is necessary to do one line of reference measurements in distilled water without the studied object for each selected scanning plane. In the designed measurement set-up such lines can be quickly registered, before submerging the object, for all 1024 planes of scanning around the object with an angular step of $\sim 0.351^{\circ}$. Additionally, amplitudes, times and frequencies of the pulses registered in water for the reference line make it possible to correct measurements in relation to differences in parameters of elementary piezoelectric transducers of the circular array and minor deviations in their correct positioning on the inner circle of the array's ring.

\section{Measurements}

The designed research stand (Fig. 3) was utilized to perform ultrasonic projection measurements of threedimensional women's breast phantoms used for ultrasonography assisted biopsy training. The reconstructed images showing parallel projection of three different acoustic parameters in the objects' structure: propagation velocity $c_{p}(x, z)$, attenuation $\alpha_{p}(x, z)$ and derivative of ultrasonic wave attenuation in relation to frequency $\partial \alpha_{p}(x, z) / \partial f$, were obtained on the basis of a set of registered received pulses with resolution of $\Delta x=\Delta s \approx 0.7 \mathrm{~mm}$ (after transformation), $\Delta z=1 \mathrm{~mm}$. The measurements were performed for 257 chords and a $79 \mathrm{~mm}$ vertical section range. Figure 8 (in rainbow colours) and Fig. 9 (in greyscale) present a set of projection images of those 3 parameters for one of the studied objects in a selected pro-

a)

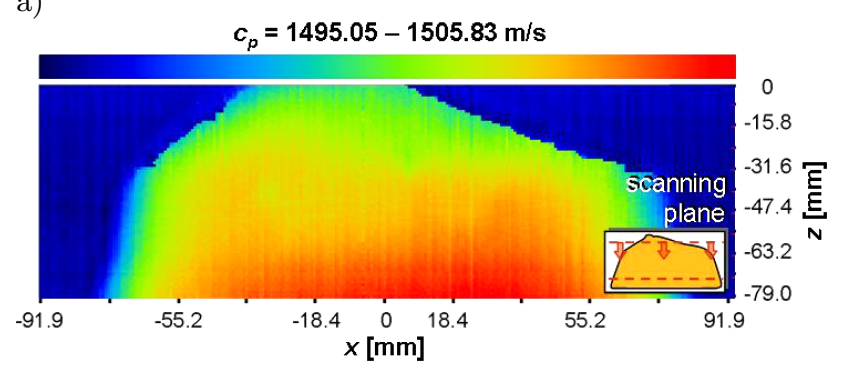

b)

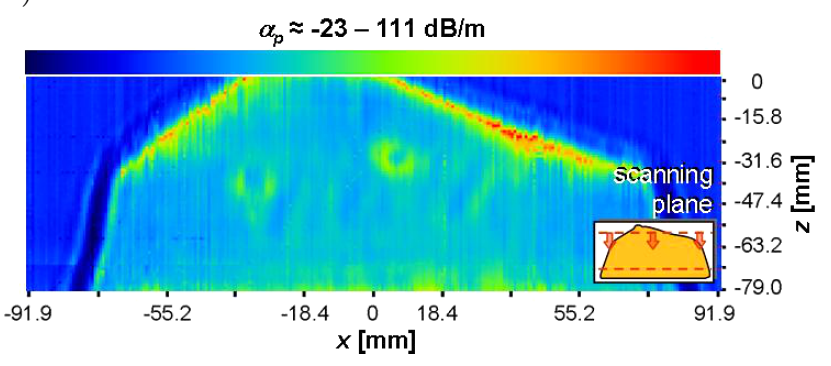

c)

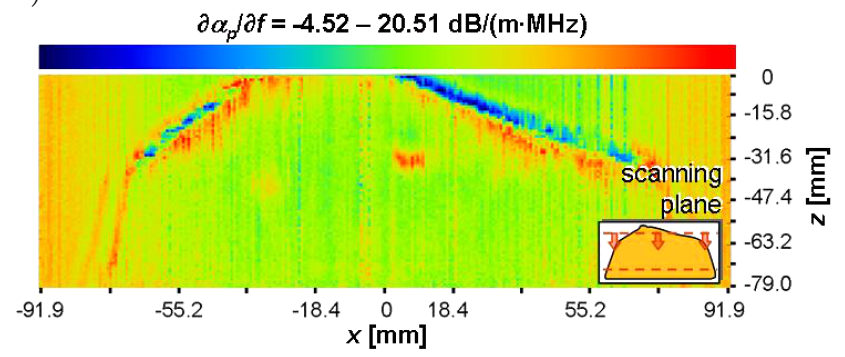

Fig. 8. Ultrasonic projection images of the studied biopsy CIRS Model 052A breast phantom (rainbow colours, contrast $50 \%$, brightness $50 \%$ ) submerged in water, determined on the basis of the measurements of: a) ultrasonic wave pulse runtime - ultrasound velocity image, b) amplitude of the ultrasonic wave pulse after it passed through - ultrasound attenuation image, c) downshift of the mid frequency of ultrasonic wave pulse after it passed through - image of derivative of ultrasound attenuation in relation to frequency. 
a)

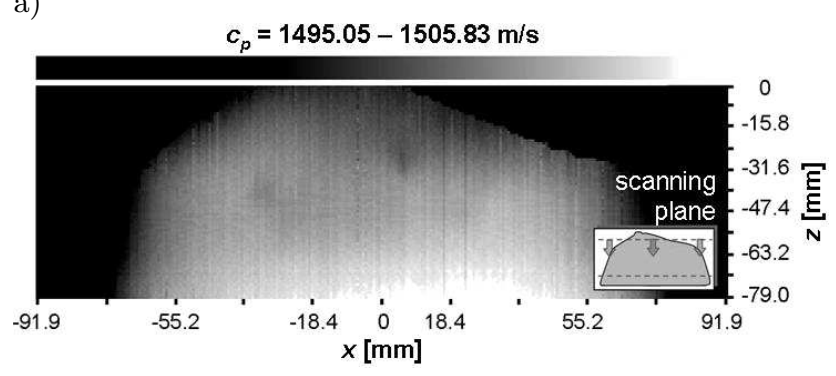

b)

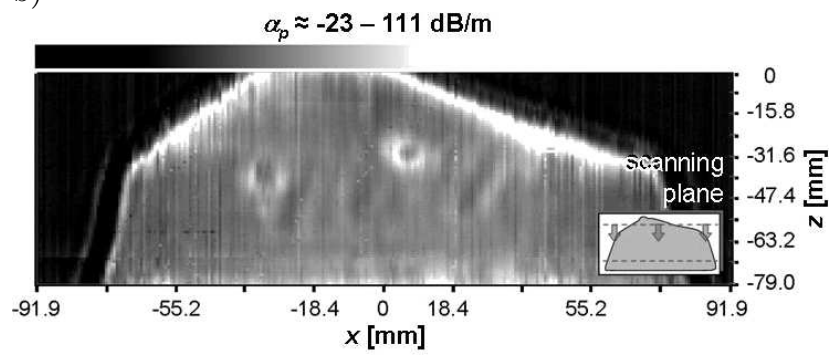

c)

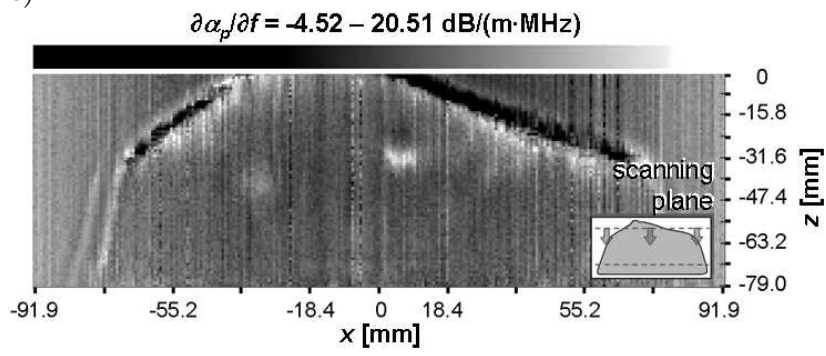

Fig. 9. Ultrasonic projection images of the studied biopsy CIRS Model 052A breast phantom submerged in water, determined on the basis of the measurements of: a) ultrasonic wave pulse runtime - ultrasound velocity image (greyscale, contrast $70 \%$, brightness $40 \%$ ), b) amplitude of the ultrasonic wave pulse after it passed through - ultrasound attenuation image (greyscale, contrast $80 \%$, brightness $60 \%$ ), c) downshift of the mid frequency of ultrasonic wave pulse after it passed through - image of derivative of ultrasound attenuation in relation to frequency (greyscale, contrast $70 \%$, brightness $40 \%$ ).

jection plane (along the longest side) - a CIRS Model $052 \mathrm{~A}$ breast phantom submerged in a tank with distilled water (used as a coupling medium).

The phantom was placed on a stand in a way that simulates supine body position (the base was perpendicular to the surface of the transducers). According to manufacturer's specifications, the studied phantom has 6 amorphous (not spherical in shape) $8-15 \mathrm{~mm}$ green inclusions that imitate cysts and 6 amorphous 6 $12 \mathrm{~mm}$ black inclusions that imitate hard lumps. The position of the inclusions in the phantom is random. One of the advantages of the phantom, in relation to its use for ultrasonic transmission studies in water, is its smooth surface which minimizes attenuation of oblique incident ultrasonic wave.

Negative values of pixels in projection images of attenuation and derivative of attenuation in relation to frequency result from the errors in measurements of pulse amplitude and spectrum after it passes near the edges of the structures, where signal loss and dropout often occurs due to diffraction and refraction.

In order to obtain reference 3-D structure of the studied CIRS Model 052A phantom, it was measured by means of the standard computerized tomography method (CT) using X-rays with resolution of $\Delta x=$ $\Delta y=0.52 \mathrm{~mm}, \Delta z=0.63 \mathrm{~mm}$. During measurements the phantom was positioned in a way that simulates standing body position (the base was parallel to the gantry). From the layered images in coronal planes that were obtained using multiplanar reconstruction (MPR) it was possible to acquire images in sagittal planes. Based on them, the shapes of all the separate heterogeneities in the phantom's structure were rendered in Fig. 10, by means of placing them over one another in the order in which they appear in the images from front to back sections.

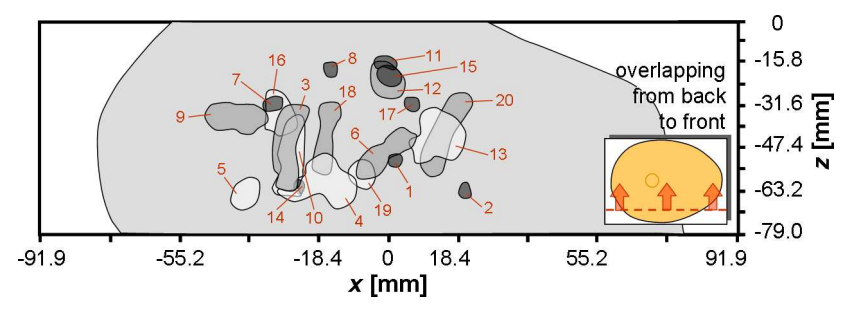

Fig. 10. Shapes of all the separate heterogeneities in the structure of the examined CIRS Model 052A breast phantom, rendered from $\mathrm{CT}$ images in sagittal projection.

Inclusions characterised by lower X-ray attenuation in comparison to phantom gel (cysts) are marked with dark grey, while those characterised by higher attenuation (lumps) are marked with light grey. It was also discovered that the structure of the phantom had air pockets (marked with black). Additionally, in order to simulate mammographic images of the phantom's structure without mechanical compression, Fig. 11 presents overlapping CT images (from back to front sections and the other way round) with $90 \%$ transparency and enhanced contrast, reconstructed in particular sagittal sections. As a result, overlapping heterogeneities in sagittal projection are visible with various transparency level, depending on the order in which the images were stacked. It is important to note that the CT method is not used in medical diagnostics for women's breast examination because of radiation beam rigidity (usually $120 \mathrm{keV}$ ), which in turn results in too low differences in radiation attenuation in tissues. For the same reason the CT images of the CIRS Model 059 breast phantom structure reconstructed from direct measurements using energy of $80 \mathrm{keV}$ or $140 \mathrm{keV}$ did not allow recognition of heterogeneous areas. Inclusions in the phantom gel were only visualised on secondary reconstructions of images of dual-energy CT examination (WANG, PelC, 2011) 


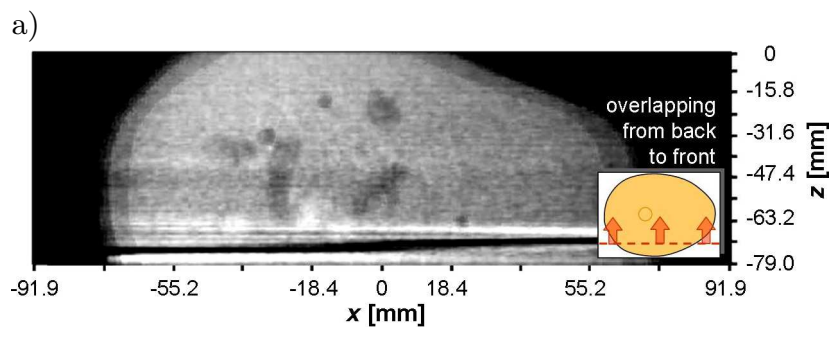

b)

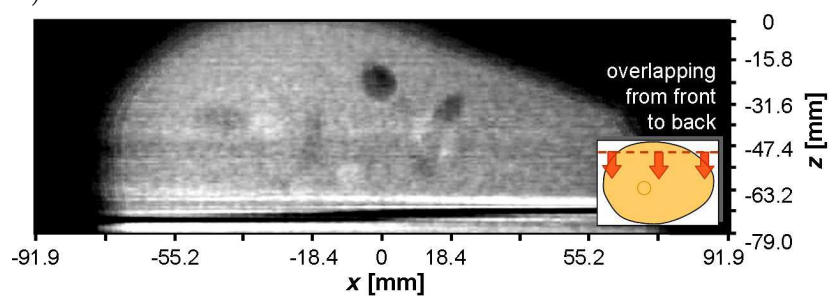

Fig. 11. Simulation of X-ray mammography in the form of overlapping CT images reconstructed by means of MPR method in individual sagittal sections, with $90 \%$ transparency and $80 \%$ contrast: a) sections stacked from back to front, b) sections stacked from front to back.

(energy of $40 \mathrm{keV}$ ). Using this method it is possible to simulate an enhanced mammographic image without the need to mechanically compress the phantom as this would cause damage.

\section{Image analysis}

When doing comparative analysis of ultrasonic and $\mathrm{X}$-ray images it is necessary to consider the movement of inclusions in the structure of the soft gel resulting from uneven phantom deformation during projection tests in supine (Fig. 8, Fig. 9) and standing body position (Fig. 10, Fig. 11). Deformation of the phantom is also caused by buoyancy force in water.

Projection image of ultrasound velocity distribution (Fig. 8a, Fig. 9a) predominantly makes it possible to detect in the background heterogeneous areas of the velocity values which are lower than the surrounding (cysts) by as little as a single $[\mathrm{m} / \mathrm{s}]$. Since the projection method is used for imaging (signal parameter values in projections increase/decrease with larger object size along the wave beam propagation path), heterogeneities for which the velocity values are slightly higher than the surrounding (compact lumps) are much more difficult to observe. One of the disadvantages of this imaging solution is that it produces fuzzy edges which may result in errors in evaluation of the size of inclusions.

Projection image of the distribution of attenuation coefficient indirectly visualises continuous and stepped changes (Fig. 8b, Fig. 9b). It is a good complement of projection images of velocity and ultrasound attenuation derivative as it is usually characterised by significant diversification of values of attenuation coeffi- cient in inclusions, in comparison to the background. Looking at the image, it is possible (especially with increased contrast) to identify heterogeneities overlapping in a projection (Fig. 9b). The image shows air pockets as light pixels. This is similar to the appearance of errors resulting from projection measurements on the phantom edges. Projection values of ultrasound attenuation coefficient are distorted as a result of weakening of the transmitted signal caused by beam divergence, propagation through layered structures, multiple reflections and existence of air bubbles.

Projection image of distribution of derivative of the ultrasound attenuation coefficient in relation to frequency (Fig. 8c, Fig. 9c) is better for visualising edges than continuous changes. However, the derivative values are distorted because received pulses are overlapped by side and multiple reflections and interference, and as a result of assuming linear changes of ultrasound attenuation with frequency. This image, however, allows the size of heterogeneous areas to be estimated in a more effective way, than projection image of ultrasound velocity (compare Fig. 8a with Fig. 8c and Fig. 9a with Fig. 9c).

Projection images (especially of the contrast type Fig. 9) clearly show vertical bands caused by stepped diversification of projection values for successive parallel measurement rays (transmitter-receiver pairs) which results from errors introduced during transformation related to equalising lengths and mutual distances between those rays.

The quality of projection images obtained using circular ultrasonic transducer array can be further enhanced by improving the construction of the array in terms of ring production precision, positioning of elementary transducers and by aligning their parameters through better selection. Additionally, it is possible to improve the precision of measurements and calculations of projection values and design smart algorithms for elimination of characteristic interference from projection images when processing data and images.

\section{Conclusions}

An analysis of the obtained results shows that the three visualised distributions of the projection values of acoustic parameters of biological media structure: ultrasound velocity $c_{p}(x, z)$, ultrasound attenuation coefficient $\alpha_{p}(x, z)$ and derivative of the ultrasound attenuation coefficient in relation to frequency $\partial \alpha_{p}(x, z) / \partial f$, are good complementary to one another (compare Fig. 8 and Fig. 9 with Fig. 10 and Fig. 11).

A 1024-element circular ultrasonic transducer array produces scanning resolution of about $0.7 \mathrm{~mm}$. Measurements are fast, because transmitting and receiving transducers are electronically switched, and the array is mechanically moved only vertically. This solution minimises production costs of the array and electronic 
components and guarantees sufficient ultrasonic wave intensity thanks to the option of adjusting the active surface of the elementary transducers by increasing their height. Additionally, same as in ultrasonographic transducer arrays (USG probes), it is possible to use horizontal phase focusing. In this case, however, it is necessary to analyse the effect of nonlinear phenomena (WóJCIK, 2004) have on introduction of artefacts in projection images. Reducing the beam's vertical width can be realised in a similar way as in USG probes, using specially shaped lens on the transducer ring or with an aperture 1.5-D or 1.75-D (WILDES et al., 1997).

Ultrasonic projection imaging method that utilizes a circular ultrasonic transducer array can be applied in medicine for diagnostic examination of women's breast as so-called ultrasonic mammography. However, in comparison to X-ray mammography, it eliminates the need to expose patients to the harmful ionising radiation.

\section{References}

1. Brettel H., Roeder U., Scherg C. (1981), Ultrasonic transmission camera for medical diagnosis, Biomedizinische Technik, 26, p.63.

2. Chino R.Y., Thomas L.J. (1996), Aperture formation on reduced-channel arrays using the transmit-receive apodization matrix, 1996 IEEE Ultrasonics Symposium Proceedings, 1567-1571.

3. Drinkwater B.W., Wilcox P.D. (2006), Ultrasonic arrays for non-destructive evaluation: A Review, NDT\&E International, 39, 525-541.

4. Duck F.A. (1990), Physical Properties of Tissue A Comprehensive Reference Book, Academic Press, London, Chap. 4, 73-135.

5. Duric N., Littrup P., Poulo L., Babkin A., Pevzner R., Holsapple E., Rama O., Glide C. (2007), Detection of breast cancer with ultrasound tomography: First results with the Computed Ultrasound Risk Evaluation (CURE) prototype, Medical Physics, 34, 2, 773-785.

6. Eames M.D.C., Hossack J.A. (2008), Fabrication and evaluation of fully-sampled, twodimensional transducer array for "Sonic Window" imaging system, Ultrasonics, 48, 376-383.

7. Ermert H., Keitmann O., Oppelt R., Granz B., Pesalento A., Vester M., Tillig B., Sander V. (2000), A New Concept For A Real-Time Ultrasound Transmission Camera, IEEE Ultrasonics Symposium Proceedings, San Juan, 1611-1614.

8. Granz B., Oppelt R. (1987), A Two Dimensional PVDF Transducer Matrix as a Receiver in an Ultrasonic Transmission Camera, Acoustical Imaging, 15, Plenum Press, New York, 213-225.

9. Green P.S., Schaefer L.F., Jones E.D., Suarez J.R. (1974), A New High Performance Ultrasonic Camera, Acoustical Holography, 5, Plenum Press, New York, 493-503.
10. Gudra T., Opielinski K.J. (2006), The ultrasonic probe for the investigating of internal object structure by ultrasound transmission tomography, Ultrasonics, 44, e679-e683.

11. Hoctor R.T., Kassam S.A. (1990), The Unifying Role of the Coarray in Aperture Synthesis for Coherent and Incoherent Imaging, Proceedings of the IEEE, 78, 4, 735-752.

12. Johnson J.A., Karaman M., Khuri-Yakub B.T. (2005), Coherent-Array Imaging Using Phased Subarrays. Part I: Basic Principles, IEEE Transactions on Ultrasonics, Ferroelectrics, and Frequency Control, 52, $1,37-50$.

13. KaK A.C., Slaney M. (1988), Principles of Computerized Tomographic Imaging, IEEE Press, New York, Chap. 3, 49-111.

14. Karaman M., Wagant I.O., Oralkan Ö., KhurIYAKUB B.T. (2009), Minimally Redundant 2-D Array Design for 3-D Medical Ultrasound Imaging, IEEE Transactions on Medical Imaging, 28, 7, 1051-1061.

15. Kim J-J., Song T-K. (2006), Real-Time High-Resolution 3D Imaging Method Using 2D Phased Arrays Based on Sparse Synthetic Focusing Technique, 2006 IEEE Ultrasonics Symposium Proceedings, 1995-1998.

16. Landini L., Sarnelli R., Squartini F. (1985), Frequency-Dependent Attenuation in Brest Tissue Characterization, Ultrasound in Medicine and Biology, 11, 4, 599-603.

17. OpIELIŃsKi K.J. (2011), Application of transmission waves for characterization and imaging of biological media structures [in Polish], Oficyna Wydawnicza Politechniki Wrocławskiej, Wrocław.

18. Opielinski K.J. (2012), Ultrasonic Projection, Chap. 1 in Ultrasonic Waves, A. Dos Santos Junior [Ed.], InTech, Rijeka, 29-58.

19. Opielinski K.J., Gudra T. (2004), Three-dimensional reconstruction of biological objects' internal structure heterogeneity from the set of ultrasonic tomograms, Ultrasonics, 42, 1-9, 705-711.

20. Opielinski K.J., Gudra T. (2005), Computer Recognition of Biological Objects' Internal Structure Using Ultrasonic Projection, [in:] Computer Recognition Systems, M. Kurzyński, E. Puchała, M. Woźniak, A. Żołnierek [Eds.], Springer-Verlag, Berlin, 645-652.

21. Opielinski K.J., Gudra T. (2010), Multielement ultrasonic probes for projection imaging of biological media, Physics Procedia, 3, 1, 635-642.

22. Opielinski K.J., GudRA T. (2013), Ultrasonic projection imaging of biological media, Proc. of Meetings on Acoustics, 19, Acoustical Society of America, 075008.

23. Opieliński K.J., Gudra T., Pruchnicki P. (2010a), A Digitally Controlled Model of an Active Ultrasonic Transducer Matrix for Projection Imaging of Biological Media, Archives of Acoustics, 35, 1, 75-90.

24. Opieliński K.J., Gudra T., Pruchnicki P. (2010b), Narrow Beam Ultrasonic Transducer Matrix Model for Projection Imaging of Biological Media, Archives of Acoustics, 35, 1, 91-109. 
25. Opieliński K.J., Gudra T., Pruchnicki P., Podgórski P., Kraśnicki T., Kurcz J., SAcisiaDEK M. (2013), Ultrasound Transmission Tomography Imaging of Structure of Breast Elastography Phantom Compared to US, CT and MRI, Archives of Acoustics, 38, 3, 321-334.

26. Opielinski K.J., Pruchnicki P., Gudra T. (2011), 2-D Directional Ultrasonic Passive Matrix of $512 \mathrm{El}$ ementary Transducers for Projection Imaging of Biological Media, [in:] International Congress on Ultrasonics Gdańsk 2011 - AIP Conference Proceedings, B.B.J. Linde, J. Pączkowski, N. Ponikwicki [Eds.], American Institute of Physics, New York, 1433, 199202.

27. Reguieg D., Padilla F., Defontaine M., Patat F., Laugier P. (2006), Ultrasonic Transmission Device Based on Crossed Beam Forming, IEEE Ultrasonics Symposium Proceedings, Vancouver, 2108-2111.

28. Wang A.S., PelC N.J. (2011), Synthetic CT: Simulating low dose single and dual energy protocols from a dual energy scan, Medical Physics, 38, 10, 5551-5562.
29. Wildes D.G., Chiao R.Y., Daft Ch.M.W., Rigby K.W., Smith L.S., Thomenius K.E. (1997), Elevation performance of $1.25 D$ and $1.5 D$ Transducer Arrays, IEEE Transactions on Ultrasonics, Ferroelectrics, and Frequency Control, 44, 5, 1027-1037.

30. WóJCIK J. (2004), Nonlinear reflection and transmission of plane acoustic waves, Archives of Acoustics, 29, $4,607-632$.

31. Wygant I.O., Lee H., Nikozadeh A., Yeh D.T., Oralkan Ö., Karaman M., Khuri-Yakub B.T. (2006), An Integrated Circuit with Transmit Beamforming and Parallel Receive Channels for Real-Time Three-Dimensional Ultrasound Imaging, 2006 IEEE Ultrasonics Symposium Proceedings, 2186-2189.

32. Yang J.N., Murphy A.D., Madsen E.L., ZagzebSKi J.A., Gilchrist K.W., Frank G.R., MCDOnald M.C., Millard C.A., Faraggi A., JaramilLo C.A., Gosset F.R. (1991), A Method for in Vitro Mapping of Ultrasonic Speed and Density in Breast Tissue, Ultrasonic Imaging, 13, 91-109. 